\title{
SpeakUp in Class!
}

Anonymous Temporary Social Media for Better Interactions

A. Holzer*, S. Govaerts*, A. Vozniuk*, B. Kocher**, D. Gillet* - *EPFL \& **UNIL, Lausanne, Switzerland

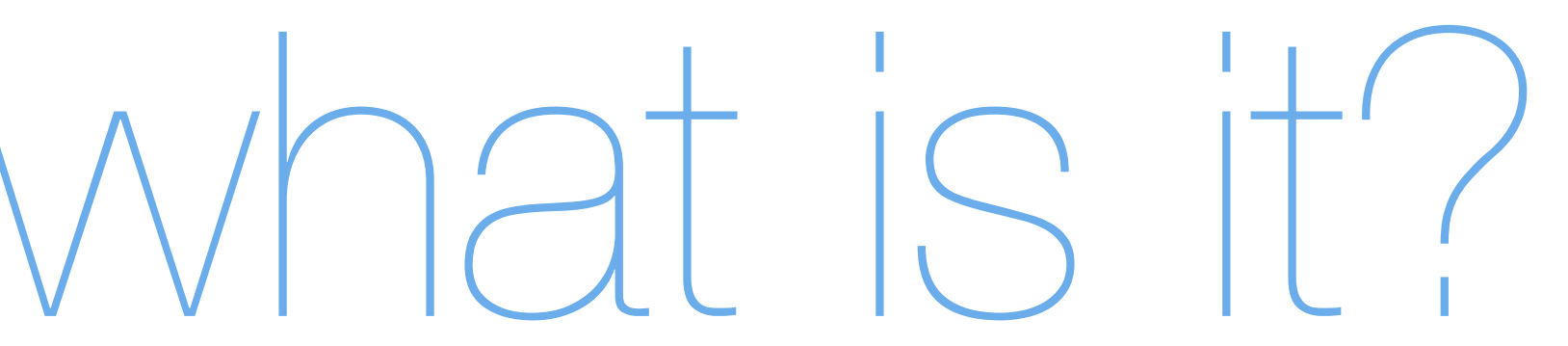

SpeakUp lets the audience anonymously share and rate each other's questions in a room. The speaker can then easily answer the best ones.

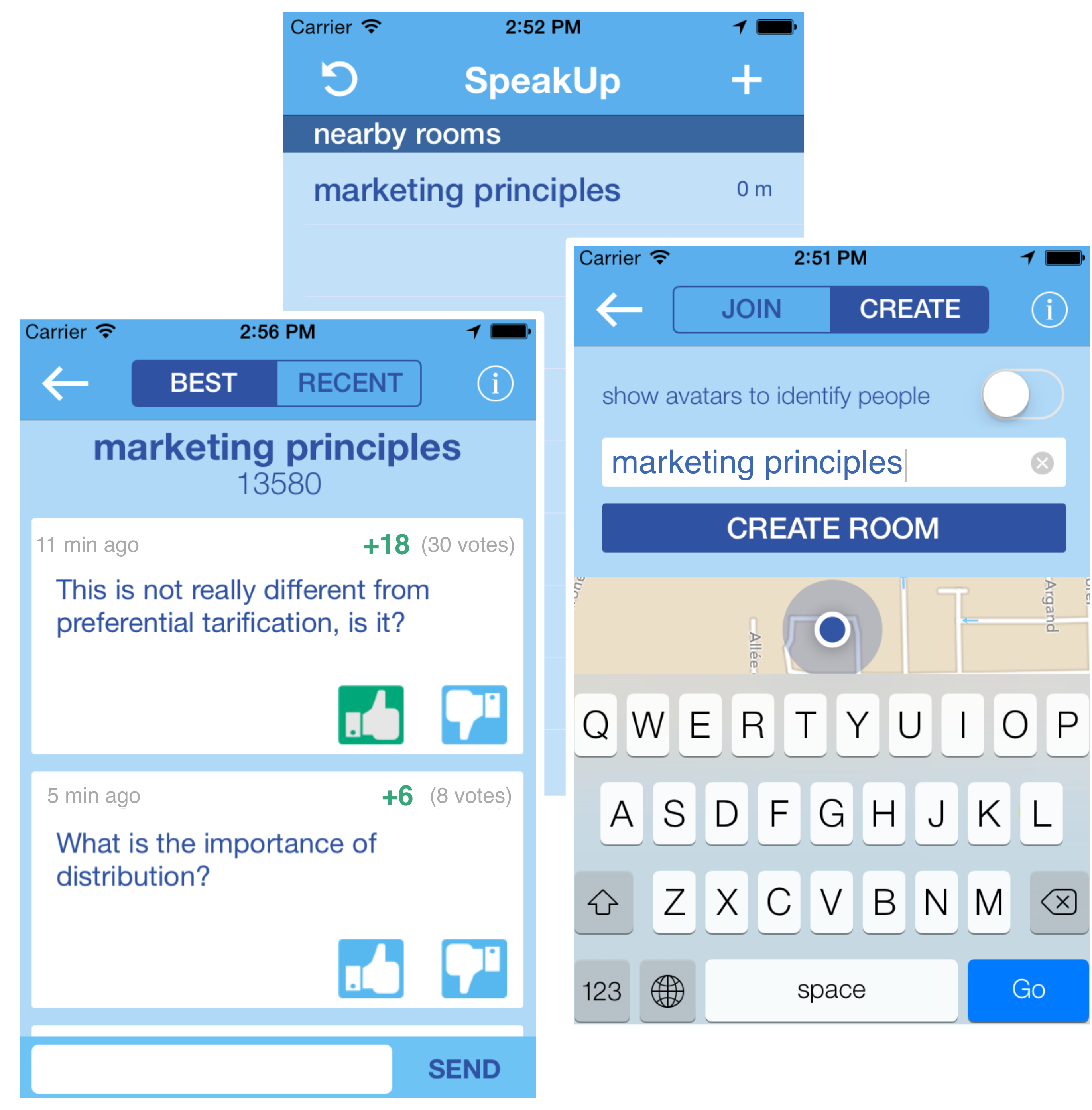

\section{temporary privacy ++}

Posting public messages online contrasts with the sense of privacy of a classroom. Like classrooms, SpeakUp chatrooms are localized. Only people nearby can find and access them. Like interactions in a classroom, messages do disappear after a while.

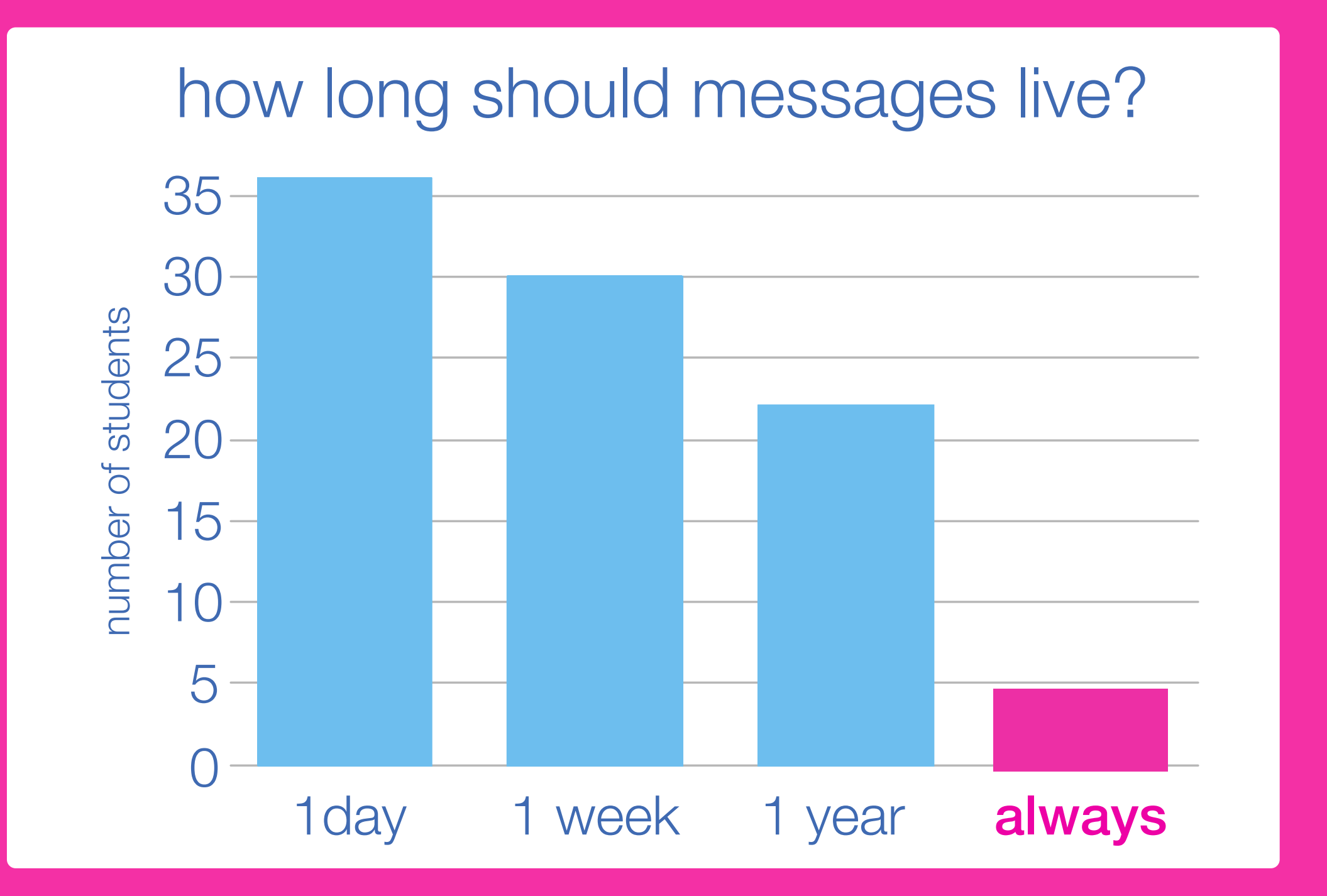

\section{social quality +}

Asking questions without knowing what others think makes it difficult to know if the question is useful to them. Through message rating it is possible to assess the

interestingness. Although

interestingness can differ among

students and the teacher, who

might perceive some as spam.

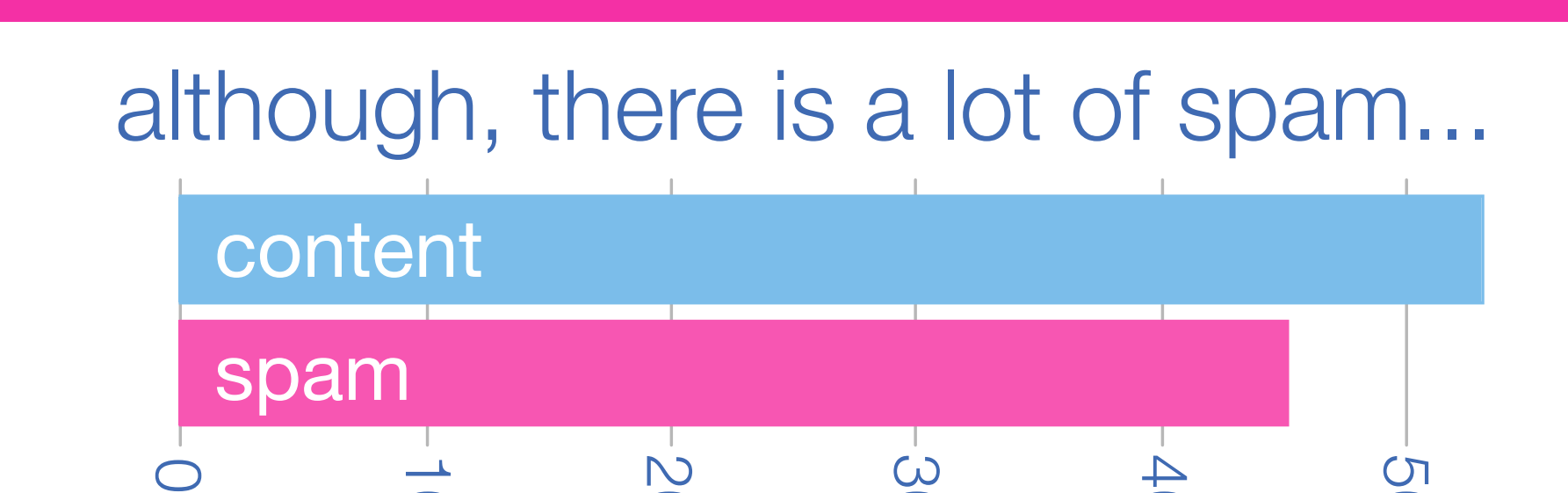

the asked questions are interesting.

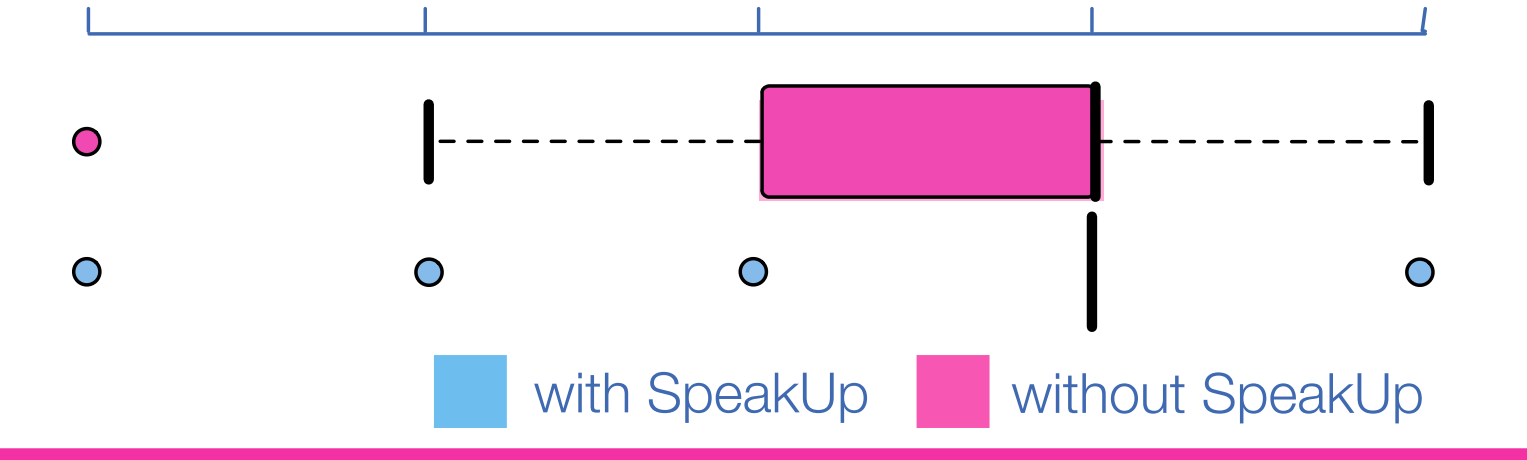

\section{anonymous quantity ++}

Speaking in front of a large

classroom is intimidating. SpeakUp

encourages students to post

messages, they can do so

completely anonymously, with no

need to log in.

\section{evaluation}

with over 300 bachelor

business students split in a

control and a SpeakUp group

$300+$

4

during 6 sessions of the

principles of marketing course

lasting 3.5 hours each

$21 h$

$\longrightarrow$

we conducted a survey after

three weeks and one at the

end, totalling 223 responses.

223

[?

over all the sessions, 222

messages were posted

222
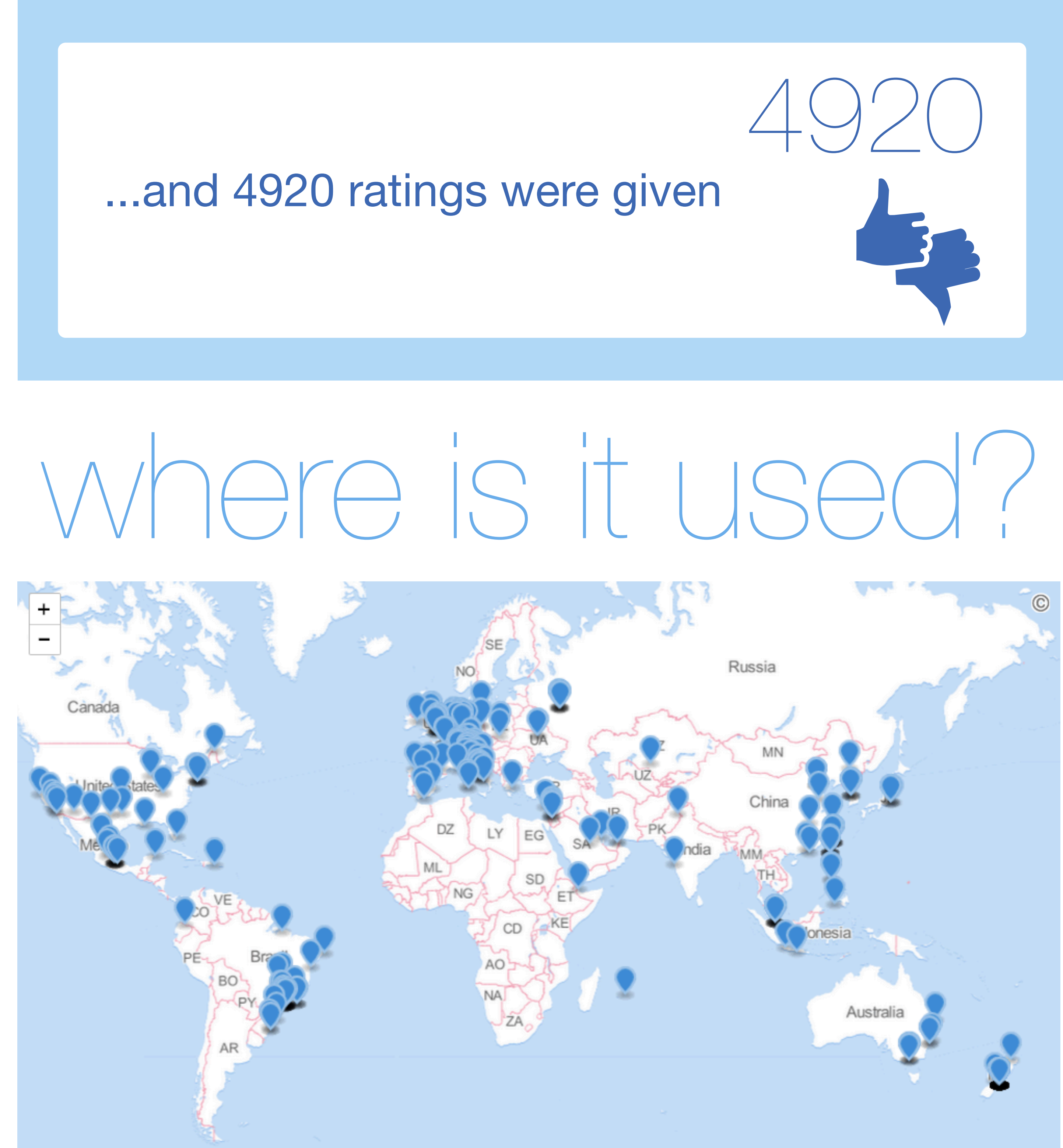

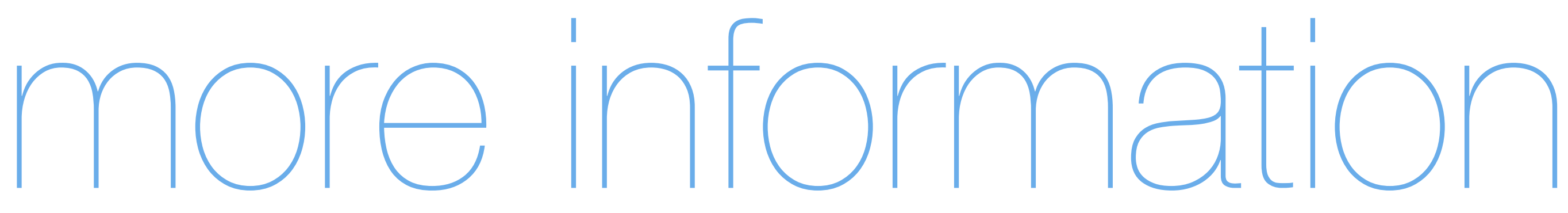

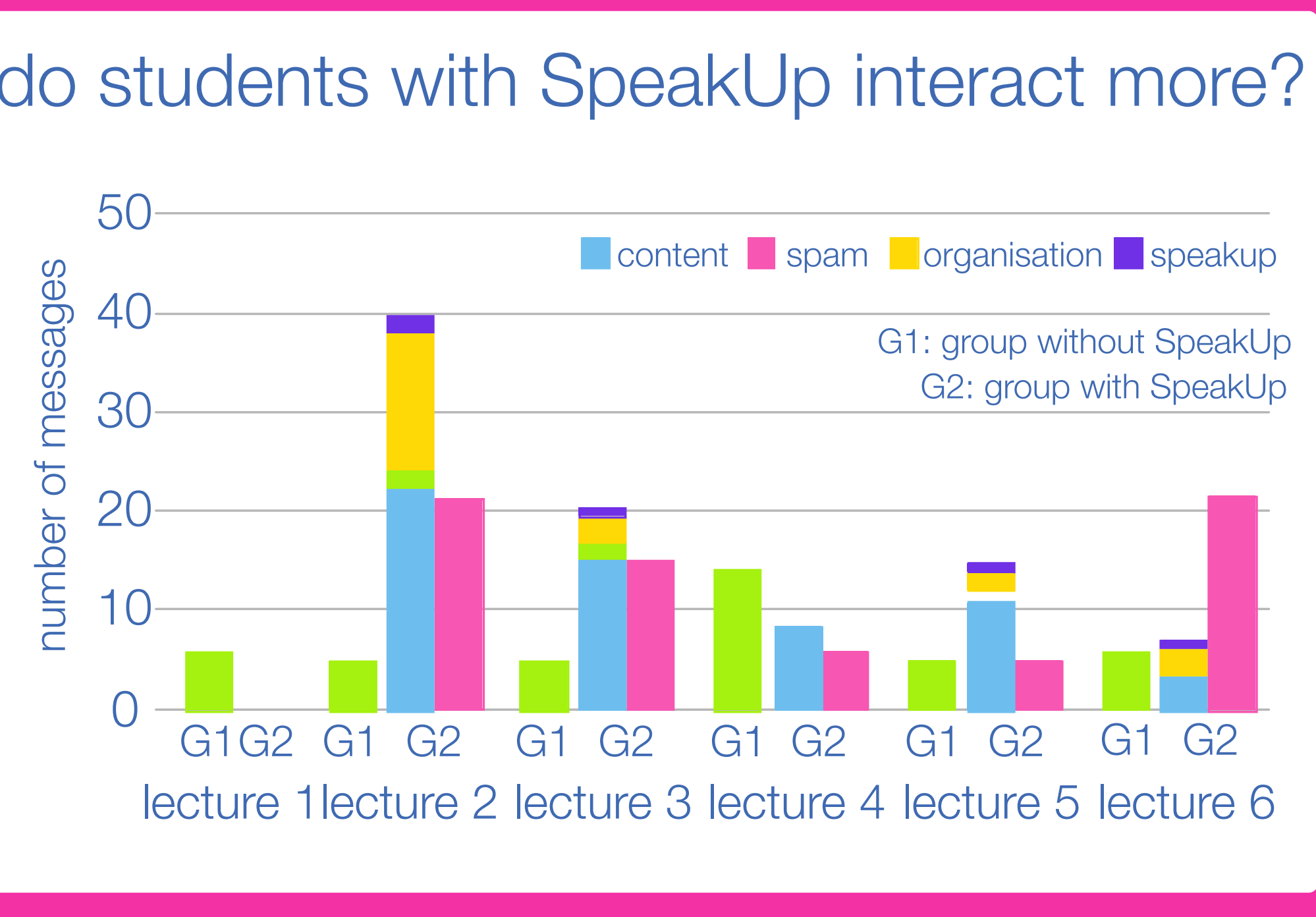

(1) App Store

Google play

( 